\title{
Unraveling Acetylcholine Impact on Human Cortical Plasticity
}

\author{
Antonio Suppa ${ }^{1}$ and Odysseas Papazachariadis ${ }^{2}$ \\ ${ }^{1}$ IRCCS Neuromed Institute and ${ }^{2}$ Department of Physiology and Pharmacology, "Sapienza” University of Rome, 00185 Rome, Italy \\ Review of Grundey et al.
}

In recent years, a growing number of studies on humans have used repetitive transcranial magnetic stimulation (rTMS) techniques to induce and examine plasticity in the primary motor cortex (M1). This plasticity is reflected by long-term changes in motor-evoked potential (MEP) amplitudes elicited by single-pulse TMS over M1 (after-effects; Ziemann et al., 2008). The techniques include paired associative stimulation (PAS), which consists of TMS delivered over M1 after electrical stimulation of nerves in the contralateral wrist. If TMS is delivered $25 \mathrm{~ms}$ after nerve stimulation $\left(\mathrm{PAS}_{25}\right)$, it induces a long-term increase in MEP size; if it is delivered $10 \mathrm{~ms}$ after nerve stimulation $\left(\mathrm{PAS}_{10}\right)$, it reduces MEP size (Stefan et al., 2000). Since PAS involves repetitive activation of sensorimotor circuits and since the direction of after-effects strictly depends on the specific interstimulus intervals used, PAS is considered to elicit a Hebbian form of heterotopic spike timingdependent plasticity (Stefan et al., 2000; Ziemann et al., 2008).

Another experimental approach used to elicit cortical plasticity is transcranial direct current stimulation (TDCS). When the anode is placed over M1 and the cathode over the contralateral frontal pole (anodal TDCS), MEPs increase in size;

Received May 21, 2012; revised June 14, 2012; accepted June 20, 2012.

Correspondence should be addressed to Dr. Antonio Suppa, IRCCS Neuromed Institute and Department of Neurology and Psychiatry, Sapienza University of Rome, Viale dell'Università, 30, 00185 Rome, Italy. E-mail: antonio.suppa@uniroma1.it.

DOI:10.1523/JNEUROSCI.2441-12.2012

Copyright $\odot 2012$ the authors $\quad 0270-6474 / 12 / 3210795-02 \$ 15.00 / 0$ when polarity is inverted (cathodal TDCS), MEPs decrease in size (Nitsche and Paulus, 2000). TDCS works through whole-brain polarization and is believed to induce depolarization or hyperpolarization of the resting membrane potential (Nitsche and Paulus, 2000). Both PAS- and TDCS-induced aftereffects are believed to reflect long-term potentiation (LTP)- and long-term depression (LTD)-like mechanisms (Nitsche and Paulus, 2000; Stefan et al., 2000; Ziemann et al., 2008).

Recent studies have investigated the possibility of boosting PAS- and TDCSinduced plasticity using drugs, including those that act on cholinergic transmission (Kuo et al., 2007; Thirugnanasambandam et al., 2011; Grundey et al., 2012). Acetylcholine (ACh) may play an important role in attention, learning, and memory by modulating cortical LTP and LTD (Laviolette and van der Kooy, 2004; Changeux, 2010). After enhancing global cholinergic transmission with rivastigmine, a cholinesterase inhibitor, Kuo et al. (2007) found that anodal TDCS failed to increase MEPs, whereas $\mathrm{PAS}_{25}$ elicited long-term MEP enhancement. More recently, Thirugnanasambandam et al. (2011) examined the specific contribution of the nicotinic ACh receptor (AChR) to PAS- and TDCSinduced plasticity in nonsmokers. Nicotine patches reduced the expected $\mathrm{PAS}_{10^{-}}$and cathodal TDCS-induced MEP inhibition. In addition, anodal TDCS left MEP amplitudes unchanged, whereas $\mathrm{PAS}_{25}$ induced long-term MEP facilitation. Unlike TDCS, $\mathrm{PAS}_{25}$ is believed to induce "focal plasticity" by activating specific sensory afferent in- puts. The authors of those studies consequently hypothesized a "focusing effect" of ACh on cortical plasticity (Kuo et al., 2007; Thirugnanasambandam et al., 2011). This hypothesis is consistent with experimental studies demonstrating that the activation of muscarinic and nicotinic AChRs presynaptically inhibit cortical recurrent glutamatergic synapses and facilitate excitatory synapses driven by afferent fibers. Thus, ACh may have a focusing effect in humans, increasing the signal-to-noise ratio of cortical information processing (Laviolette and van der Kooy, 2004; Kuo et al., 2007; Changeux, 2010)

It is important to assess the effects exerted by nicotine on plasticity not only in nonsmokers but also in smokers. This topic was addressed in a recent study published in The Journal of Neuroscience (Grundey et al., 2012). Grundey et al. (2012) applied PAS and TDCS in smokers with mild nicotine dependence after $10 \mathrm{~h}$ of nicotine withdrawal. In subjects under nicotine withdrawal conditions (placebo patch), PAS $_{10}$ and cathodal TDCS elicited the expected inhibitory MEP changes, whereas $\mathrm{PAS}_{25}$ and anodal TDCS failed to elicit MEP facilitation. Grundey et al. (2012) ascribed the reduced LTP-like plasticity to nicotinic AChR desensitization due to chronic nicotine consumption. In contrast, $\mathrm{PAS}_{25^{-}}$and anodal TDCS-induced MEP facilitation were restored, and even prolonged, in subjects under nicotine patch conditions. Overall, these findings are in agreement with the deficit-compensating hypothesis of nicotine consumption, i.e., nicotine restores brain 
functions impaired by nicotine withdrawal (Laviolette and van der Kooy, 2004; Changeux, 2010; Grundey et al., 2012). In addition, $\mathrm{PAS}_{10}$ induced inhibitory MEP changes, whereas cathodal TDCS does not. Grundey et al. (2012) interpreted the overall impact of nicotine on cortical plasticity as a nicotine-induced focusing effect. Under nicotine, $\mathrm{PAS}_{25}$ was hypothesized to enhance MEP facilitation because PAS involves afferent sensory inputs to the M1, whereas TDCS does not (Kuo et al., 2007; Thirugnanasambandam et al., 2011; Grundey et al., 2012).

Although the hypothesis of a nicotineinduced focusing effect on plasticity is consistent with the increased responses to $\mathrm{PAS}_{25}$, it does not explain the reduced responses to $\mathrm{PAS}_{10}$. In addition, the results of Grundey et al. (2012) are consistent with those of Swayne et al. (2009), who observed that nicotine enhanced "focal" LTP-like plasticity elicited by intermittent theta-burst stimulation (iTBS). However, iTBS involves applying rhythmic gamma bursts $(50 \mathrm{~Hz})$ at theta frequencies $(5 \mathrm{~Hz})$ over M1 without activating afferent sensory inputs (Ziemann et al., 2008).

We believe another mechanism may contribute to the nicotine-induced focusing effect. The threshold required to induce LTP/LTD varies according to the history of synaptic activity, and plasticity can be inhibited, boosted, or stabilized according to homeostatic metaplasticity processes (Ziemann and Siebner, 2008). Experimental studies have demonstrated that acute nicotine exposure increases the activity of GABA interneurons (Laviolette and van der Kooy, 2004; Changeux, 2010). In nonsmokers, acute nicotine challenge enhances responses to $\mathrm{PAS}_{25}$ and reduces re- sponses to $\mathrm{PAS}_{10}$ and cathodal TDCS, possibly as a result of homeostatic metaplasticity mechanisms triggered by increased GABA activity. The reduced responses to anodal TDCS are more likely to reflect the focusing effect of nicotine reported by previous studies (Kuo et al., 2007; Thirugnanasambandam et al., 2011). Conversely, chronic exposure to nicotine alters the balance between inhibitory and excitatory interneurons, induces nicotinic AChR upregulation, and decreases GABA activity because of AChR desensitization (Laviolette and van der Kooy, 2004; Changeux, 2010). Thus, the LTP-like plasticity due to reduced $\mathrm{PAS}_{25}$ and anodal TDCS in chronic smokers under nicotine withdrawal conditions might reflect homeostatic metaplasticity mechanisms triggered by decreased GABA activity. Our hypothesis is in agreement with the observation that acute nicotine challenge in smokers under nicotine withdrawal conditions restores, at least in part, the physiological boundaries of cortical plasticity. However, it should be borne in mind that nicotinic AChR desensitization may not persist after $>10 \mathrm{~h}$ of nicotine withdrawal and that desensitization varies according to the specific AChR subtypes ( $\alpha_{7}$ or $\alpha_{4} \beta_{2}$ receptors).

In conclusion, the study by Grundey et al. (2012) provides an important insight into the cholinergic modulation of cortical plasticity in humans. Definite and straightforward conclusions cannot, however, be easily drawn because of the heterogeneous impact of ACh on cortical function. The varying responses to plasticity-inducing protocols used in smokers and nonsmokers should be taken into account as a possible confounding effect when future studies are planned.

\section{References}

Changeux JP (2010) Nicotine addiction and nicotinic receptors: lessons from genetically modified mice. Nat Rev Neurosci 11:389401.

Grundey J, Thirugnanasambandam N, Kaminsky K, Drees A, Skwirba AC, Lang N, Paulus W, Nitsche MA (2012) Neuroplasticity in cigarette smokers is altered under withdrawal and partially restituted by nicotine exposition. J Neurosci 32:4156-4162.

Kuo MF, Grosch J, Fregni F, Paulus W, Nitsche MA (2007) Focusing effect of acetylcholine on neuroplasticity in the human motor cortex. J Neurosci 27:14442-14447.

Laviolette SR, van der Kooy D (2004) The neurobiology of nicotine addiction: bridging the gap from molecules to behaviour. Nat Rev Neurosci 5:55-65.

Nitsche MA, Paulus W (2000) Excitability changes induced in the human motor cortex by weak transcranial direct current stimulation. J Physiol 527:633-919.

Stefan K, Kunesch E, Cohen LG, Benecke R, Classen J (2000) Induction of plasticity in the human motor cortex by paired associative stimulation. Brain 123:572-584.

Swayne OB, Teo JT, Greenwood RJ, Rothwell JC (2009) The facilitatory effects of intermittent theta burst stimulation on corticospinal excitability are enhanced by nicotine. Clin Neurophysiol 120:1610-1615.

Thirugnanasambandam N, Grundey J, Adam K, Drees A, Skwirba AC, Lang N, Paulus W, Nitsche MA (2011) Nicotinergic impact on focal and non-focal neuroplasticity induced by non-invasive brain stimulation in nonsmoking humans. Neuropsychopharmacology 36:879-886.

Ziemann U, Siebner HR (2008) Modifying motor learning through gating and homeostatic metaplasticity. Brain Stimul 1:60-66.

Ziemann U, Paulus W, Nitsche MA, PascualLeone A, Byblow WD, Berardelli A, Siebner HR, Classen J, Cohen LG, Rothwell JC (2008) Consensus: motor cortex plasticity protocols. Brain Stimul 1:164-182. 